\title{
Oral treatment with an antioxidant (raxofelast) reduces oxidative stress and improves endothelial function in men with Type II diabetes
}

\author{
P.J. Chowienczyk ${ }^{1}$, S. E. Brett ${ }^{1}$, N.K. Gopaul ${ }^{2}$, D.Meeking ${ }^{3}$, M. Marchetti ${ }^{4}$, D. L. Russell-Jones ${ }^{3}$, E. E. Änggård ${ }^{2}$, \\ J.M. Ritter ${ }^{1}$ \\ ${ }^{1}$ Department of Clinical Pharmacology, Centre for Cardiovascular Biology and Medicine, King's College, London, UK \\ ${ }^{2}$ The William Harvey Research Institute, St Bartholomew's Hospital Medical College, London, UK \\ ${ }^{3}$ Department of Diabetes and Endocrinology, King's College, London, UK \\ ${ }^{4}$ Biomedica Foscama, Rome, Italy
}

\section{Abstract}

Aims/hypothesis. To determine whether raxofelast, a new water soluble antioxidant decreases oxidative stress and improves endothelial function in men with Type II (non-insulin dependent) diabetes mellitus.

Methods. We treated ten normotensive, normocholesterolaemic men with Type II diabetes and as controls ten healthy men matched with them for age with raxofelast (600 mg twice daily) for 1 week. Plasma 8-epi-PGF ${ }_{2 \alpha}$, a non-enzymic oxidation product of arachidonic acid was measured by gas chromatography/mass spectrometry as an index of oxidative stress. Forearm vasodilator responses to brachial artery infusion of acetylcholine $(7.5,15$ and $30 \mu \mathrm{g}$ $\mathrm{min}^{-1}$ ) and of the nitric oxide donor nitroprusside (1, 3 and $10 \mu \mathrm{g} \mathrm{min}^{-1}$ ) were measured by strain gauge plethysmography.

Results. Plasma concentrations of 8-epi-PGF $\mathrm{PG}_{2 \alpha}$ were greater in diabetic than in control men $(0.99 \pm 0.20$ vs $0.18 \pm 0.01 \mathrm{nmol} \mathrm{l}^{-1}$, means $\left.\pm \mathrm{SEM}, p<0.001\right)$ and fell after raxofelast (from $0.99 \pm 0.20$ to $0.47 \pm 0.07$ nmol $\left.1^{-1}, p<0.05\right)$ in diabetic men but not in control men. Blood flow responses to acetylcholine were lower $(p<0.05)$ in diabetic than in control men $(7.4 \pm 1.0$ vs $12.9 \pm 2.3 \mathrm{ml} \cdot \mathrm{min}^{-1} \cdot 100 \mathrm{ml}^{-1}$ for the highest dose). In diabetic men, but not in control men, raxofelast increased $(p<0.05)$ blood flow responses to acetylcholine (from $7.4 \pm 1.0 \mathrm{ml} \cdot \mathrm{min}^{-1} \cdot 100 \mathrm{ml}^{-1}$ to $11.3 \pm 2.3 \mathrm{ml} \cdot \mathrm{min}^{-1} \cdot 100 \mathrm{ml}^{-1}$ at highest dose). Blood flow responses to nitroprusside were similar in control and diabetic men and in both groups were similar before and after raxofelast.

Conclusion/interpretation. Oral treatment with raxofelast for 1 week reduces oxidative stress and improves endothelial function in men with Type II diabetes. [Diabetologia (2000) 43: 974-977]

Keywords Acetylcholine, endothelium, free radicals, lipid peroxidation, nitric oxide, prostaglandins.
Type II (non-insulin dependent) diabetes mellitus is characterised by increased oxidative stress and endothelial dysfunction [1]. Both factors may have a

Received: 14 October 1999 and in revised form: 28 May 2000

Corresponding author: Dr. P.J. Chowienczyk, Department of Clinical Pharmacology, St Thomas' Hospital, Lambeth Palace Road, London SE1 7EH, UK

Abbreviations: IRFI 005, 2,3-Dihydro-5-hydroxy-4,6,7-trimethyl-2-benzofuranacetic acid; NO, nitric oxide; PG, prostaglandin major role in the initiation and development of the atherosclerotic disease which accounts for most of the excess mortality associated with Type II diabetes. Short term intra-arterial treatment with vitamin C improves endothelium-dependent vasodilation in Type II diabetes [2]. This suggests that endothelial dysfunction in Type II diabetic patients occurs secondary to increased oxidative stress, possibly as a result of reactive oxygen species such as superoxide anion inactivating endothelium-derived nitric oxide (NO). It is not known whether oral treatment with antioxidants can reduce oxidative stress in patients 
with Type II diabetes and, if so, whether this influences endothelial function. We investigated the effect of oral treatment for 1 week with raxofelast, a new hydrophilic vitamin E-like antioxidant [3] in patients with Type II diabetes and healthy control subjects. Plasma 8-epi-prostaglandin $(\mathrm{PG}) \mathrm{F}_{2 \alpha}$, a stable $\mathrm{F}_{2}$-isoprostane was measured as a marker of oxidant stress [4] and forearm blood flow responses to acetylcholine used as a measure of endothelial function.

\section{Subjects and methods}

We recruited ten men aged $47 \pm 13$ years (means \pm SD) with Type II diabetes controlled by diet alone (5/10) or by oral hypoglycaemic therapy (sulphonylureas alone in 4/10 and a sulphonylurea plus metformin in 1/10) from the St Thomas' Hospital diabetes clinic in the United Kingdom. None had clinical evidence of atherosclerosis or of microvascular complications other than mild background retinopathy $(2 / 10)$. All had urinary albumin/creatinine ratios within the normal range and none had evidence of neuropathy on vibration testing. Body mass index (BMI) was $27 \pm 5 \mathrm{~kg} \mathrm{~m}^{-2}$. Mean blood pressure was $133 \pm 15 /$ $80 \pm 8.2 \mathrm{~mm} \mathrm{Hg}$. Serum concentrations of total cholesterol and triglycerides were $4.8 \pm 1.1$ and $2.1 \pm 1.3 \mathrm{mmol} \mathrm{l}^{-1}$, respectively. Healthy control men whose ages matched with them (to within 2 years) were recruited from hospital staff. Mean values of BMI $\left(26 \pm 3 \mathrm{~kg} / \mathrm{m}^{-2}\right)$, blood pressure $(129 \pm 11 / 76 \pm$ $12 \mathrm{~mm} \mathrm{Hg})$, total cholesterol $\left(5.4 \pm 0.6 \mathrm{mmol} \mathrm{l}^{-1}\right)$ and triglycerides $\left(1.5 \pm 1.0 \mathrm{mmol} \mathrm{l}^{-1}\right)$ were similar to those in the diabetic men. Glycated haemoglobin was $8.1 \pm 2.4$ and $5.1 \pm 0.4 \%$ in diabetic and control subjects respectively $(p<0.01)$. The protocol was approved by St Thomas' Hospital Research Ethics Committee and all patients gave written informed consent. Oxidative stress and endothelial function were measured before and after treatment with raxofelast taken as capsules $(600 \mathrm{mg}$ twice daily) for 7 days. Patients omitted oral hypoglycaemic drugs on the 2 study days. Plasma concentrations of raxofelast and its active metabolite 2,3-dihydro-5-hydroxy-4,6,7-trimethyl-2-benzofuranacetic acid (IRFI 005) were measured by HPLC immediately before dosing and at 1, 2 and $4 \mathrm{~h}$ after dosing in diabetic subjects. Pharmacokinetics of raxofelast in healthy non-diabetic subjects has been reported previously [3].

Oxidative stress. Plasma 8 -epi- $\mathrm{PGF}_{2 \alpha}$ was measured by gas chromatography/negative ion mass spectrometry using deuterated $\mathrm{PGF}_{2 \alpha}$ as an internal standard [5]. In three samples peaks could not be clearly identified and data from these were not used in the statistical analysis.

Endothelial function. Endothelial function was assessed by measuring forearm blood flow responses [2] to brachial artery infusion of acetylcholine $\left(7.5,15\right.$ and $30 \mu \mathrm{g} \cdot \mathrm{min}^{-1}$, as three cumulative doses each for $5 \mathrm{~min}$ ) using strain gauge plethysmography. Nitroprusside $\left(1,3\right.$ and $\left.10 \mu \mathrm{g} \cdot \mathrm{min}^{-1}\right)$ was used as an endothelium-independent control. Subjects took the final dose of raxofelast $1 \mathrm{~h}$ before the blood flow study corresponding with peak concentrations of its active metabolite IRFI 005 [3].

Data analysis and statistical methods. Results are expressed as means \pm SEM. Forearm blood flow and concentrations of 8epi-PGF $_{2 \alpha}$ were log transformed before statistical analysis because the distribution of values thus transformed approximated a normal distribution more closely. Analysis of variance (ANOVA) for repeated measures was used to test for differ-

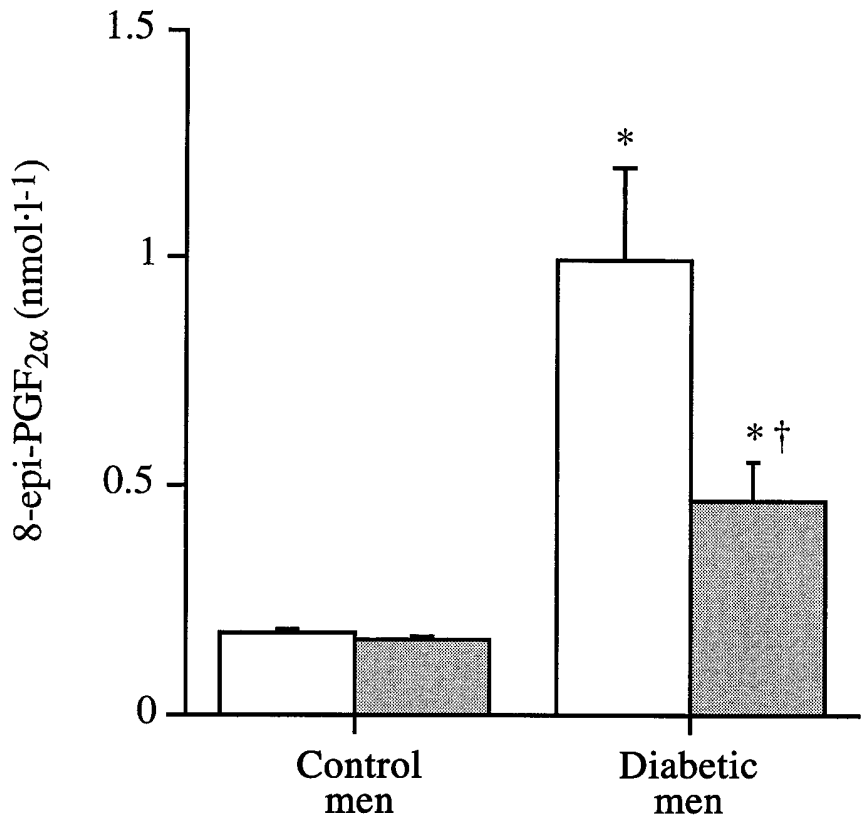

Fig. 1. Mean plasma concentrations of 8 -epi-PGF ${ }_{1 \alpha}$ before (open bars) and after (shaded bars) treatment with raxofelast in control men $(n=10)$ and men with Type II diabetes $(n=7)$. Error bars are SEMs. $* p<0.001$ compared with controls. ${ }^{\dagger} p<0.05$ compared with values before treatment

ences in plasma concentrations of 8-epi-PGF $2 \alpha$ and vasodilator responses before and after raxofelast. Paired $t$ tests were used as post-hoc tests where the ANOVA showed significant overall differences. We took $p<0.05$ as indicating significance.

\section{Results}

Plasma concentrations of raxofelast and IRFI 005. In men with diabetes, plasma concentrations of raxofelast itself both before and after treatment were below the limit of detection of the assay $\left(<0.25 \mu \mathrm{g} \cdot \mathrm{ml}^{-1}\right)$. Concentrations of the active metabolite IRFI 005 were below the limit of detection of the assay $\left(<0.25 \mu \mathrm{g} \cdot \mathrm{ml}^{-1}\right)$ before treatment. The mean trough concentration of IRFI $0051 \mathrm{~h}$ before the final dose of raxofelast was $4.1 \pm 0.7 \mu \mathrm{g} \cdot \mathrm{ml}^{-1}$. Plasma concentrations at 1 and $2 \mathrm{~h}$ after the final dose (during which time the blood flow study was undertaken) were $11.3 \pm 2.7$ and $8.8 \pm 1.3 \mu \mathrm{g} \cdot \mathrm{ml}^{-1}$, respectively. At $4 \mathrm{~h}$ after the final dose of raxofelast the mean plasma concentration of IRFI 005 was $5.3 \pm 0.8 \mu \mathrm{g} \cdot \mathrm{ml}^{-1}$.

Oxidative stress. Plasma concentrations of 8-epi$\mathrm{PGF}_{2 \alpha}$ were greater in men with Type II diabetes than in control men $(0.99 \pm 0.20$ vs $0.18 \pm 0.01$ $\left.\mathrm{nmol} \cdot \mathrm{l}^{-1}, p<0.001\right)$. After treatment with raxofelast, plasma concentrations of 8-epi- $\mathrm{PGF}_{2 \alpha}$ were reduced (Fig. $1, p<0.05$ by ANOVA). The fall was attributable to men with Type II diabetes in whom plasma concentrations fell from $0.99 \pm 0.20$ to $0.47 \pm 0.07$ $\mathrm{nmol} \cdot \mathrm{l}^{-1}(n=7, p<0.05)$. There was no significant 

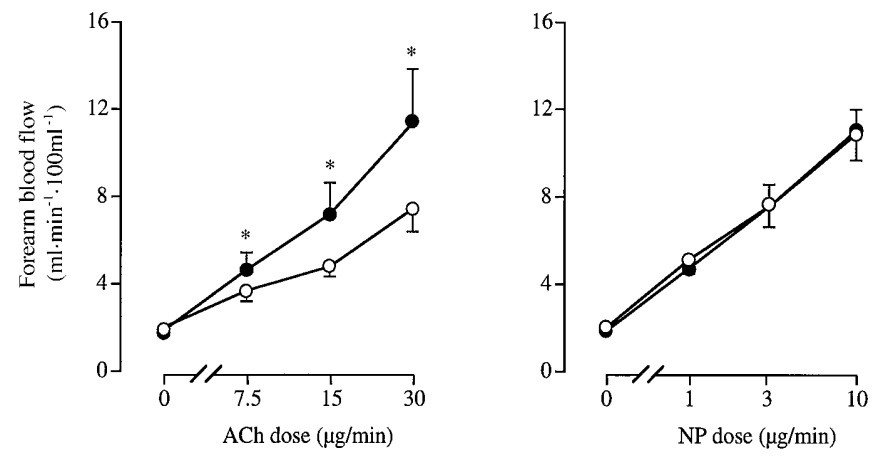

Fig. 2. Mean forearm blood flow during infusion of $\mathbf{A}$ acetylcholine (ACh) and $\mathbf{B}$ nitroprusside (NP) before (open circles) and after (closed circles) treatment with raxofelast in men with Type II diabetes $(n=10)$. Error bars are SEMs. $* p<0.05$ compared with values before treatment

change in 8-epi-PGF ${ }_{2 \alpha}$ in control subjects. Lipid profiles and fasting blood glucose did not change significantly in control men or patients with Type II diabetes after treatment with raxofelast.

Endothelial function. Mean blood flow responses to acetylcholine were lower in men with Type II diabetes than in control men. The highest dose of acetylcholine increased forearm blood flow to $12.9 \pm 2.3 \mathrm{ml} \cdot \mathrm{min}^{-1} \cdot 100 \mathrm{ml}^{-1}$ in control men and to $7.4 \pm 1.0 \mathrm{ml} \cdot \mathrm{min}^{-1} \cdot 100 \mathrm{ml}^{-1}$ in men with Type II diabetes $(p<0.05)$. In men with Type II diabetes, blood flow responses to acetylcholine after raxofelast treatment were greater than at baseline (Fig. $2, p<0.05$ ). The highest dose of acetylcholine increased blood flow to $7.4 \pm 1.0 \mathrm{ml} \cdot \mathrm{min}^{-1} \cdot 100 \mathrm{ml}^{-1}$ before and $11.3 \pm 2.3 \mathrm{ml} \cdot \mathrm{min}^{-1} \cdot 100 \mathrm{ml}^{-1}$ after treatment $(p<0.05)$. In control men responsiveness to acetylcholine was similar before and after raxofelast treatment. Blood flow responses to nitroprusside were similar in control men and in men with Type II diabetes and in both groups were similar before and after treatment.

\section{Discussion}

Increased oxidative stress in Type II diabetes could result from increased generation of reactive oxygen species or diminished antioxidant defences or both. Free radical generation could be increased as a result of glucose-induced activation of cyclo-oxygenase, autoxidation of glucose, alteration in transition metal metabolism or increased flux through the polyol pathway [1]. Cellular enzymatic antioxidants, superoxide dismutase, catalase and glutathione peroxidase and non-enzymatic antioxidants including vitamins $\mathrm{C}$ and $\mathrm{E}$ could all be depleted in Type II diabetes [6]. Lipid peroxidation is a central feature of oxidant injury and leads to the non-enzymatic formation of $\mathrm{F}_{2}$ - isoprostanes. Although 8-epi-PGF ${ }_{2 \alpha}$ cannot be regarded as a universal marker of oxidant stress (for example lipid peroxidation might not occur despite increased oxidant stress due to protection by antioxidants), it is a more sensitive marker of oxidative injury in endothelial cells than other indices such as concentrations of thiobarbituric acid reactive substances [7]. The present study confirms our previous finding that concentrations of 8 -epi-PGF $\mathrm{PG}_{2 \alpha}$ are increased in plasma from patients with Type II diabetes [5]. Oxidative stress could account, at least in part, for impaired endothelium-dependent relaxation in Type II diabetes. Reactive oxygen species such as superoxide anion inactivates endothelium-derived NO [8]. Antioxidants, particularly water soluble antioxidants, could scavenge reactive oxygen species thereby protecting NO from inactivation. The beneficial effects of improved NO availability are likely to extend beyond improved vasodilation and reduced vasospasm because NO has numerous potentially antiatherogenic actions [8].

Raxofelast and its active metabolite IRFI 005 operate by a recycling mechanism similar to the vitamin E/ascorbate system to protect LDL against oxidation [3,9]. Unlike $\alpha$-tocopherol, IRFI 005 is water soluble. It has free radical scavenging actions and, as a scavenger of superoxide anion, is more potent than non-enzymatic antioxidants such as ascorbate [3]. This scavenging activity could be particularly effective in protecting NO against inactivation by superoxide anion and thus in improving endothelial function within resistance vasculature as observed in the present study.

Our study shows that oral treatment with raxofelast for 1 week produces a pronounced reduction in oxidative stress and improves endothelial function in Type II diabetes. These findings have potential implications for use of raxofelast as a prophylactic or therapeutic agent and support the proposal that oxidative stress has a role in the endothelial dysfunction of Type II diabetes. It should be noted, however, that a dissociation between endothelial function in small mesenteric arteries of streptozotocin-diabetic rats and plasma concentrations of 8 -epi-PGF $\mathrm{PG}_{2 \alpha}$ has been observed [10]. Although this could reflect a species difference or difference between vascular beds, it is possible that raxofelast reduces 8-epi$\mathrm{PGF}_{2 \alpha}$ and improves endothelial function by different mechanisms.

Raxofelast, a new hydrophilic vitamin E-like antioxidant, reduces oxidative stress and restores endothelial function in men with Type II diabetes. The therapeutic potential of antioxidant treatment in Type II diabetes to prevent atherosclerotic disease should be pursued. 
Acknowledgement. Dr R. Centore (Biomedical Foscama) developed the HPLC assay for raxofelast and IRFI 005 and managed the assays of the study samples.

\section{References}

1. Tribe RM, Poston L (1996) Oxidative stress and lipids in diabetes: a role in endothelium vasodilator dysfunction? Vasc Med 1: 195-206

2. Ting HH, Timimi FK, Boles KS, Creager SJ, Ganz P, Creager MA (1996) Vitamin C improves endothelium-dependent vasodilation in patients with non-insulin dependent diabetes mellitus. J Clin Invest 97: 22-28

3. Campo GM, Ceccarelli S, Squadrito F, Altavilla D, Dorigotti L, Caputi AP (1997) Raxofelast (IRFI 016): a new hydrophilic vitamin E-like antioxidant agent. Cardiovasc Drug Rev 15: 157-173

4. Morrow JD, Roberts LJ (1996) The isoprostanes: current knowledge and directions for future research. Biochem Pharmacol 51: 1-9

5. Gopaul NK, Änggård EE, Mallet AI, Beteridge DJ, Wolff SP, Nourouz-Zadeh J (1995) Plasma 8-epi-PGF $2 \alpha$ levels are elevated in individuals with non-insulin dependent diabetes mellitus. FEBS Lett 368: 225-229

6. Sundaram RK, Bhaskar A, Vijayalingam S, Viswanathan M, Mohan R, Shanmugasundaram KR (1996) Antioxidant status and lipid peroxidation in Type II diabetes mellitus with and without complications. Clin Sci (Colch) 90: 255-260

7. Hart CM, Karman RJ, Blackburn TL, Gupta MP, Garcia JG, Mohler ER (1998) Role of 8-epi-PGF $2 \alpha$, 8-isoprostane, in $\mathrm{H}_{2} \mathrm{O}_{2}$-induced derangements of pulmonary artery endothelial cell barrier function. Prostaglandins Leukot Essent Fatty Acids 58: 9-16

8. Moncada S, Palmer RMJ, Higgs EA (1991) Nitric oxide: Physiology, pathophysiology, and pharmacology. Pharmacol Rev 43: 109-142

9. Iuliano L, Pedersen JZ, Camastra C, Bello V, Ceccarelli S, Violi F (1999) Protection of low density lipoprotein oxidation by the antioxidant agent IRFI005, a new synthetic hydrophilic vitamin E analogue. Free Radic Biol and Med 26: 848-868

10. Palmer AM, Gopaul N, Dhir S, Thomas CR, Poston L, Tribe RM (1998) Endothelial dysfunction in streptozotocin-diabetic rats is not reversed by dietary probucol or simvastatin supplementation. Diabetologia 41: 157-164 